\title{
Common Genetic Effects on Variation in Impulsivity and Activity in Mice
}

\author{
Anthony R. Isles, Trevor Humby, Eurof Walters, and Lawrence S. Wilkinson \\ Neurobiology and Developmental Genetics Programmes, The Babraham Institute, Babraham, Cambridge CB2 4AT, United Kingdom
}

Impulsivity is a complex psychological construct that impacts on behavioral predispositions in the normal range and has been shown to have a genetic element through the examination of hereditary patterns of abnormal conditions such as attention deficit/hyperactivity disorder and obsessive compulsive disorder. In this study, we took advantage of the isogenic nature of inbred strains of mice to determine the contribution of genes to impulsive behaviors by examining the performance of four separate mouse strains in a novel murine delayed-reinforcement paradigm, during which the animals had to choose between rewards that were relatively small but available immediately and larger but progressively delayed rewards. To control for maternal effects, all the mice were cross-fostered to a common strain immediately after birth. Under these conditions, we found significant differences between the strains on behaviors indexing impulsive choice and on independent measures of locomotor activity, which subsequent heritability analysis showed could be related, in part, to genetic effects. Moreover, the two aspects of behavior were found to co-vary, with the more active animals also displaying more impulsive behavior. This was not attributable to mundane confounds related to individual task requirements but instead indicated the existence of common genetic factors influencing variation in both impulsivity and locomotor activity. The data are discussed in terms of the coexistence of impulsivity and hyperactivity, interactions between environmental and genetic effects, and possible candidate genes.

Key words: impulsivity; activity; heritability; delayed reinforcement; mice; $\mathrm{AD} / \mathrm{HD}$

\section{Introduction}

"Impulsivity" is the term used to describe a variety of psychological processes that impact on decision making and action within the normal range and contribute to the make up of human personality (Evenden, 1999). Impulsive behavior also plays a central role in several disorders in which decision making and action might be considered pathological, including attention deficit/hyperactivity disorder (AD/HD), obsessive compulsive disorder (OCD), personality disorder, and drug addiction (Evenden, 1999; Clark and Robbins, 2002). The extent to which genetic predisposition can influence this area of psychology is uncertain, but there is some evidence for a genetic component from both linkage [e.g., in the case of AD/HD (Swanson et al., 2001)] and association (gene candidate) studies, in which, for example, a polymorphism in the gene encoding monoamine oxidase A (MAOA) has been associated with abnormally aggressive, antisocial, and criminal behavior (Brunner et al., 1993a,b). Additionally, work with animals carrying a null mutation of the $5 H T_{1 B}$ gene has demonstrated the importance of this gene product in mediating impulsive behaviors (Brunner and Hen, 1997; Bouwknecht et al., 2001; Pattij et al., 2003).

Although evidence is accruing that impulsive responding may

Received Feb. 25, 2004; revised June 16, 2004; accepted June 17, 2004.

This work was supported by the Biotechnology and Biological Sciences Research Council, UK. We thank the reviewers for helpful comments on this manuscript and Vikki Brew for help with the cross-fostering studies.

Correspondence should be addressed to Dr. Lawrence Wilkinson, Neurobiology Programme, The Babraham Institute, Cambridge CB2 4AT, UK. E-mail: lawrence.wilkinson@bbsrc.ac.uk.

DOI:10.1523/JNEUROSCI.1650-04.2004

Copyright $\odot 2004$ Society for Neuroscience $\quad$ 0270-6474/04/246733-08\$15.00/0 be an important component of psychopathological conditions, there has, to date, been little or no systematic analysis of the degree of genetic contribution to impulsivity; this is despite recent findings emphasizing the likely sensitivity of these psychological functions to selective pressures (Chen et al., 1999; Ding et al., 2002). In the present study, we exploited the isogenic nature of inbred mice, in combination with a tractable mouse model of delayed reinforcement, to look for evidence of genetic effects on impulsivity. Delayed reinforcement is one of the many facets of impulsive behavior and refers to situations in which a choice has to be made between small immediate rewards and larger, but delayed, rewards (Evenden and Ryan, 1996). The selection of smaller immediate gains in preference to larger delayed gains has been considered to reflect "impulsive" choice, whereas the opposite bias toward delayed gratification has been taken to index increasing "self-control." This aspect of psychology has been shown to be sensitive to both pharmacological manipulations (Evenden and Ryan, 1996; Cardinal et al., 2000; Isles et al., 2003) and selective brain lesions in animals (Mobini et al., 2000, 2002; Cardinal et al., 2001). Additionally, in the clinic a bias toward impulsive choice has been observed in several conditions, including AD/HD (Sonuga-Barke et al., 1992), pathological gambling (Petry, 2001), and drug addiction (Madden et al., 1997). Here, using a novel murine delayed-reinforcement task developed in our laboratory (Isles et al., 2003), we demonstrate that variation in genetic background arising from allelic polymorphisms can contribute to differences in this form of impulsive behavior. We also investigated genetic effects on locomotor activity and, in particular, the extent to which these effects covaried with impulsive responding, a question motivated by the frequently observed 
comorbidity of these two aspects of behavior (Sheppard et al., 1999). Our data indicate a significant genetic contribution as well as a positive covariance between the behaviors, the latter consistent with the action of common genetic substrates mediating variability in impulsivity and locomotor activity.

\section{Materials and Methods \\ Animals}

All animals were generated in the Small Animal Barrier Unit at The Babraham Institute. The strains used in the experiment were C57BL/6J, CBA/Ca, 129S2/SvHsd (referred to from now on as 129/Sv), and BALB/c. Pregnant parents were transferred to the Small Animal Unit (The Babraham Institute), and when the pups were born, they were cross-fostered to CD1 females that had littered the previous day. All subjects used were males aged 10 weeks and weighing $\sim 30$ gm at the outset of the experiment. The animals were housed in groups of three, four, or five under temperature-controlled conditions and under a $12 \mathrm{hr}$ light/dark cycle (lights on at 07:30 A.M.), and all behavioral testing was performed during the light phase. Standard laboratory chow was available ad libitum, but for most of the experiment, water was restricted to $2 \mathrm{hr}$ access per day (given after testing). This regime maintained the subjects at $\sim 90 \%$ of free-feeding body weight. All procedures were conducted in accordance with the requirements of the United Kingdom Animals (Scientific Procedures) Act 1986.

\section{Preference for reinforcer}

Before any experimental work, all animals were handled daily for 2 weeks, and their body weight was monitored. After this time, the animals were placed on a $20 \mathrm{hr}$ water-restriction schedule for $4 \mathrm{~d}$ and then on a 22 $\mathrm{hr}$ water restriction for an additional $10 \mathrm{~d}$ until body weight had stabilized. The animals were then habituated to the liquid reinforcer used in the operant procedures (10\% solution of condensed milk; Nestle, Croydon, UK). Habituation was performed outside the operant chambers as described previously (Humby et al., 1999). Briefly, the subjects were given four separate $10 \mathrm{~min}$ sessions over $5 \mathrm{~d}$ with an excess of either water or the reinforcer presented in two small bowls (apart from the first session in which two samples of water were presented). Habituated levels of reinforcer preference (ratio of the volume of milk consumed to the volume of water consumed), and the total volumes drunk, were assessed on day 5 of testing.

\section{Delayed-reinforcement task}

Impulsivity was measured in an operant delayed-reinforcement paradigm performed in operant chambers modified for use with mice. The behavioral and pharmacological validation of the task was described previously (Isles et al., 2003). The mice were trained to respond with a nose-poke to one of two identical visual stimuli that differed only in location; one response resulted in a small quantity of reinforcer, the other in a larger quantity of reinforcer (see Fig. 1). As the session proceeded, increasing delay was introduced onto the response leading to the large reinforcer. Hence, impulsive choice was operationally defined as the extent to which the mice switched responding from the larger, but progressively, delayed reinforcer to a response that provided a smaller amount of reinforcer immediately.

Shaping. Initial shaping was performed over 20 sessions. The first five sessions involved general habituation, panel pressing, and learning that food was available in the magazine. In sessions $6-12$, the subject was required to make a contingent nose-poke in the center hole in response to a $10 \mathrm{sec}$ light stimulus to initiate food delivery. In the final eight sessions, the subject had to respond sequentially to $10 \mathrm{sec}$ light stimuli presented first in the central location and then (pseudo-randomly) either to the left or right of the center before food was delivered. In this way, the center nose-poke acted as a cue, signaling the beginning of a trial and focusing the behavior of the mice toward the subsequent presentation of stimuli in the lateral locations. The animals then moved on to the delayedreinforcement task proper in which, critically, in the choice component of the task, stimuli in the lateral holes were presented simultaneously.

Delayed reinforcement. The delayed-reinforcement task comprised five sequential blocks of eight trials in which a nose-poke response in one

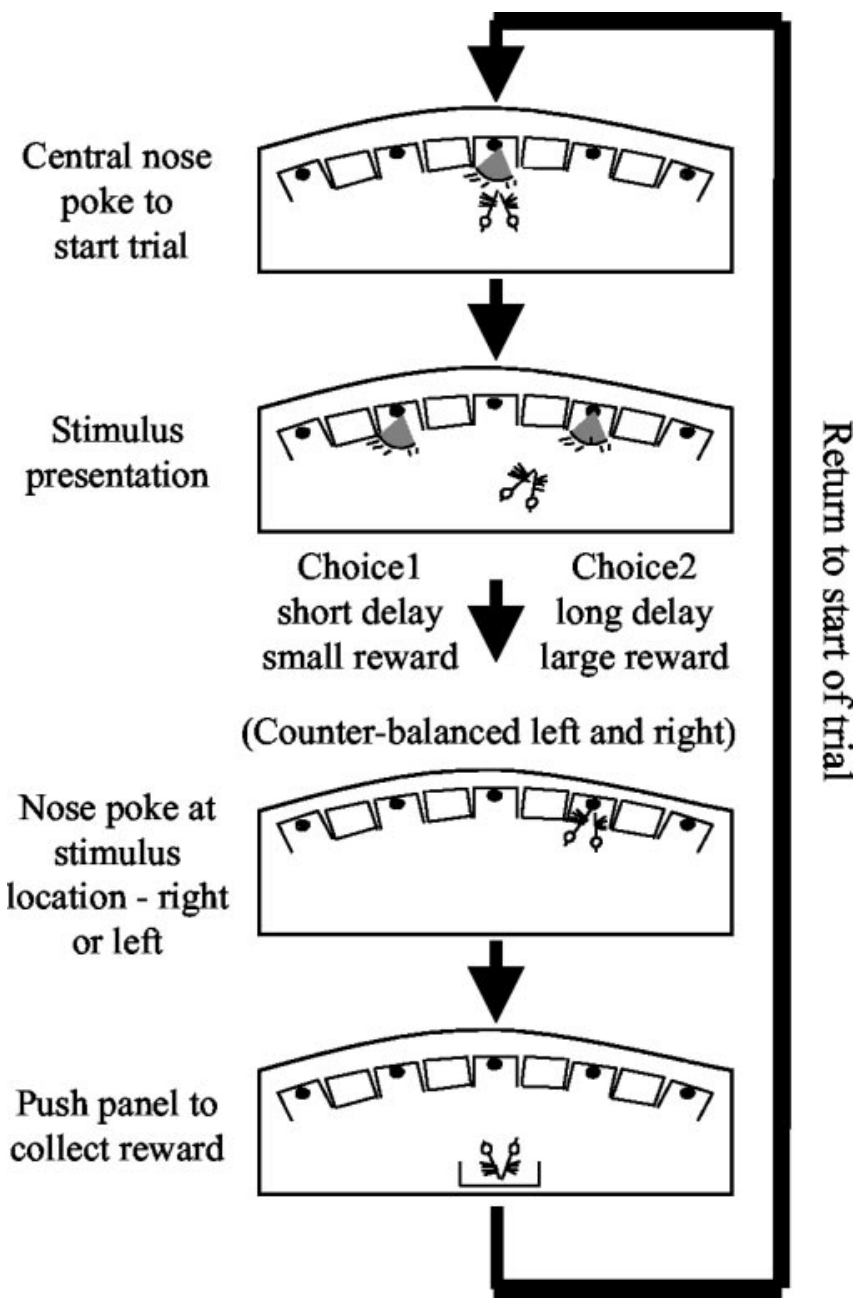

Figure 1. Delayed-reinforcement task for mice. Schematic diagram showing the configuration of the nine-hole box and the basic sequence of responses made by the mouse in a choice trial of the delayed-reinforcement task.

direction, left or right, resulted in the delivery of $50 \mu \mathrm{l}$ of reinforcer, with a response in the other direction resulting in the delivery of $25 \mu \mathrm{l}$ of reinforcer. The large and small response directions were kept constant for each mouse but were counterbalanced between subjects. In block 1, either response led to the immediate delivery of reinforcer. In subsequent blocks, increasing delays were introduced between the response and the delivery of the larger reinforcer. Initially, the mice were trained on delay pairs of the following (small reward response first): 0 sec versus 0 sec; 0 sec versus $0.5 \mathrm{sec}$; $0 \mathrm{sec}$ versus $1 \mathrm{sec} ; 0$ sec versus $2 \mathrm{sec}$; and $0 \mathrm{sec}$ versus 4 $\mathrm{sec}$ for $10 \mathrm{~d}$. They were then moved on to the full-delay range: $0 \mathrm{sec}$ versus $0 \mathrm{sec} ; 0 \mathrm{sec}$ versus $1 \mathrm{sec}$; $0 \mathrm{sec}$ versus $2 \mathrm{sec}$; $0 \mathrm{sec}$ versus $4 \mathrm{sec}$; and $0 \mathrm{sec}$ versus $8 \mathrm{sec}$ to stable baseline performance (see below for baseline performance criteria). Trials 1 and 2 in any block were "forced" information trials, whereby after the central nose-poke only one of the two response options was available. This was designed to provide the subjects with prior notice of the extent of any delay associated with the larger reward response. In the next six trials, the animals had a choice of either right or left as shown in Fig. 1. Both stimuli were presented for $10 \mathrm{sec}$ each and were extinguished once an animal had made a nose-poke. No central nose-poke at the start of a trial was recorded as a "non-started trial," and a no choice nose-poke was recorded as an "omission." Regardless of the outcome of a trial, a new trial was started $45 \mathrm{sec}$ after the presentation of the previous central stimulus. Keeping the length of the task constant ensured that the rate of delivery of reinforcement associated with both behavioral responses was identical, preventing any differences influencing choice. The food-hopper light was illuminated when the reinforcer 
was delivered, however, there was no light or programmed signal during the delayed period between the choice nose-poke and delivery of the food.

\section{Task manipulations}

Once stable baseline performance had been achieved, a series of manipulations of the basic task parameters were performed to assess the extent to which choice behavior was controlled specifically by delay and was independent of satiation effects. As described previously (Isles et al., 2003), these manipulations consisted of probe conditions in which zero delay persisted on the large reward response throughout a session (zero delay) and reversal of delay order $(8,4,2,1$, and $0 \mathrm{sec})$. In all cases, stable baseline conditions were reestablished between the task manipulations.

\section{Locomotor activity}

Before testing in the delayed-reinforcement task, the spontaneous locomotor activity levels of the four different strains of mice were measured for 3 consecutive days, using a battery of activity cages fitted with infrared beams as described by Humby et al. (1999). The activity cages consisted of clear Perspex boxes $(210 \times 210 \times 365 \mathrm{~mm})$ containing two transverse infrared beams $10 \mathrm{~mm}$ from the base, spaced equally along the length of the box. The number of beam breaks and runs (scored as consecutive breaks of each beam) during the $120 \mathrm{~min}$ sessions were recorded in $5 \mathrm{~min}$ bins by a computer. All sessions were run under red-lighting conditions.

\section{Statistics and heritability analysis}

The preliminary data assessing initial reinforcer preference/habituation were analyzed by ANOVA with the factor "strain" (C57BL/6J, 129/Sv, $\mathrm{CBA} / \mathrm{Ca}, \mathrm{BALB} / \mathrm{c})$. Data from the delayed-reinforcement task were analyzed by ANOVA with the factors strain, "delay" $(0,1,2,4$, and $8 \mathrm{sec})$, "choice" (small/immediate or larger/delayed choice), and "direction" (ascending or descending delays condition). Stable baseline performance in the delayed-reinforcement task was defined as when data from three consecutive sessions showed significant effects of delay but no effect of "session" (baseline sessions 1, 2, and 3), i.e., to meet baseline criteria, performance of the four strains had to be delay dependent and stable over three sessions. Activity data were analyzed by ANOVA with the factors strain and "days" (days 1, 2, and 3 of consecutive testing). Tukey's post hoc tests were performed where appropriate.

Estimates of heritability and genetic correlations between the measures of impulsivity and activity were computed from the components of variance and covariance derived from hierarchical analyses of variance and covariance, as described by Hegmann and Possidente (1981). The analysis provided estimates of the genetic (between-strains variability) and nongenetic (within-strain variability) contribution to the behaviors and then assessed the extent to which they covaried. Two behavioral measures of impulsivity were used in the analysis, the average choice bias across the session and choice bias at the longest delay. In both cases, the behavioral measures were taken at stable baseline performance of the delayedreinforcement task. Variability in activity levels between the strains was assessed using the data from day 3 of locomotor activity testing.

A typical ANOVA would take the following form:

Equation 1

\begin{tabular}{llll}
\hline Source of variation & $\begin{array}{l}\text { Degrees of } \\
\text { freedom }\end{array}$ & Mean square & $\begin{array}{l}\text { Expected } \\
\text { mean square }\end{array}$ \\
\hline $\begin{array}{l}\text { Between strains } \\
\text { Within strains }\end{array}$ & 3 & $A$ & $\sigma^{2}+k \sigma_{\mathrm{s}}^{2}$ \\
Total & $\mathrm{N}-4$ & $B$ & $\sigma^{2}$ \\
\hline
\end{tabular}

where the constant $k$ represents the degree of replication in a balanced arrangement but is a function of the unequal replicate numbers in unbalanced arrangements. The components of variance for strain and environment are denoted by $\sigma_{\mathrm{S}}{ }^{2}$ and $\sigma^{2}$, respectively, and may be derived, quite simply, from the ANOVA. Thus, $\sigma^{2}$ is estimated directly as $B$, whereas $\sigma_{\mathrm{S}}{ }^{2}$ is estimated as $(A-B) / k$.

Following Hegmann and Possidente (1981), we defined the heritability of inbred strains as follows: Heritability $={\sigma_{\mathrm{S}}}^{2} /\left({\sigma_{\mathrm{S}}}^{2}+2 \sigma^{2}\right)$. Heritability, as defined here, is a simple function of the $F$ ratio $(A / B)$, which would be used to test the strain effect in the ANOVA displayed above. Thus, Heritability $=(F-1) /(F+2 k-1)$.

To estimate the genetic and environmental correlations, the outline ANOVA displayed above needs to computed for the impulsivity data, for the activity data, and for the cross product of activity and impulsivity. If we denote the strain components of variance for impulsivity and activity by ${\sigma_{\mathrm{Si}}}^{2}$ and $\sigma_{\mathrm{Sa}}{ }^{2}$, respectively, and the component of covariance by $\sigma_{\mathrm{Sia}}$, the genetic correlation coefficient is estimated as follows: $r_{\mathrm{g}}=\sigma_{\mathrm{Sia}}$ l $\sqrt{ }\left[{\sigma_{\mathrm{Si}}}^{2} \times{\sigma_{\mathrm{Sa}}}^{2}\right]$, with a similar type of expression for the environmental correlation.

The sampling distribution of both heritability and genetic correlations is likely to be asymmetric so that the SEs of these statistics are of limited value in making informed inferences. However, because heritability is a simple function of the variance ratio $(F)$, we converted confidence limits for the $F$ statistic into the corresponding limits for heritability.

Confidence limits for an observed value of the variance ratio $(F)$ statistic may be estimated using the non-central $F$ distribution. Unfortunately, tables of this distribution were not easily accessible, in that they did not appear in the familiar volumes of statistical tables. Several excellent approximations have been proposed, however (Pearson and Tiku, 1970; Stuart and Ord, 1991), and these were used to derive confidence limits in the present study. The test of whether an estimate of heritability is statistically significant was based on whether these confidence limits embrace the null hypothesis value of zero.

Similar problems applied when making inferences about genetic correlation estimates. As we have already noted, the SEs quoted in the literature (Falconer and Mackay, 1996) may not be useful. Furthermore, there appears to be no useful direct correspondence with other (tabulated) statistics, as in the case of heritability. We therefore developed a Monte Carlo simulation program to derive the sampling distribution of both the heritability estimates and of the correlation coefficients (genetic and environmental). This routine can provide confidence limits on the statistics of interest, and the test of significance is again based on whether these limits embrace the null hypothesis value (which is zero for both heritability and the correlation coefficients).

All statistical analyses were performed using GenStat release 6.1.

\section{Results}

At the outset of the experiment, there was a total of 35 animals; however, four animals (two 129/Sv and two CBA/Ca) developed an intractable side bias in the delayed-reinforcement task and so were excluded from all other analyses.

\section{Reinforcer preference}

All four strains of mice showed an initial and equivalent preference for the condensed milk reinforcer used to motivate performance in the delayed-reinforcement task (Fig. 2) (no main effects of strain; $F_{(3,27)}=0.89$; NS). The total volumes of fluid drunk (milliliters) were as follows: C57BL/6J, $1.3 \pm 0.3 \mathrm{ml} ; 129 / \mathrm{Sv}$, $2.2 \pm 0.2 \mathrm{ml}$ CBA/Ca, $1.5 \pm 0.2 \mathrm{ml}$ BALB/c, $1.7 \pm 0.2 \mathrm{ml}$.

\section{Strain differences in choice bias}

Choice behavior of the four inbred strains in the delayedreinforcement task at stable baseline levels of performance is shown in Figure $3 A$. Baseline performance criteria were achieved by all four strains after seven sessions on the full-delay range. The data shown are the means of the next three sessions. All four groups exhibited a systematic change in choice bias away from the response leading to the delayed (larger) reinforcer toward the immediate (smaller) reinforcer with increasing delay (main effect of delay; $\left.F_{(4,135)}=7.5 ; p<0.001\right)$. However, the choice bias functions were different across the groups (main effect of strain; $\left.F_{(3,135)}=14.7 ; p<0.001\right)$, with the BALB/c and C57BL/6J mice exhibiting a pattern consistent with a relatively greater degree of impulsive choice and the 129/Sv and CBA/Ca strains exhibiting a pattern consistent with a relatively lesser degree of impulsive 


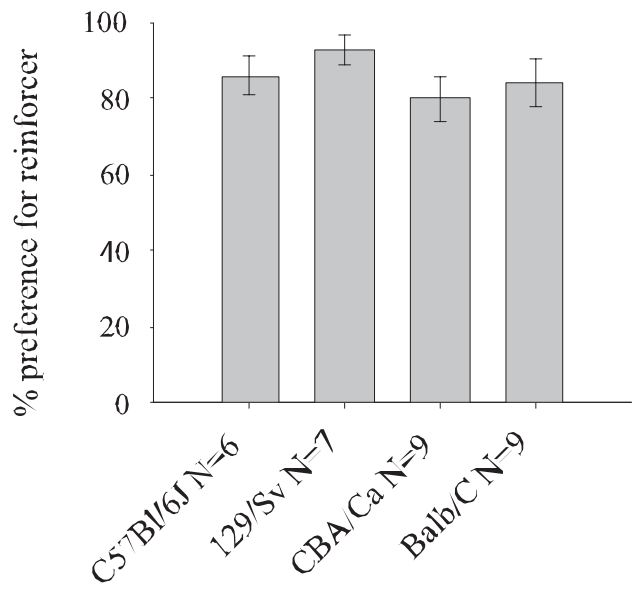

Figure 2. Preference for reinforcer across strains. Graph showing equivalent preference across strains for the milk reinforcer on day 5 of the preference test assessed before the delayedreinforcement task. Data shown are means \pm SEM.

choice. Post hoc tests confirmed the following, in terms of impulsive choice: $\mathrm{C} 57 \mathrm{BL} / 6 \mathrm{~J}=\mathrm{BALB} / \mathrm{c}>129 \mathrm{~Sv}=\mathrm{CBA} / \mathrm{Ca}$. The between-strain variation in impulsive choice was manifest across all delays, as confirmed by the lack of interaction between strain and delay $\left(F_{(12,135)}=0.30 ; \mathrm{NS}\right)$. The pattern of strain effects was stable and persisted throughout the experiment, as illustrated in Figure $3 B$, which shows the mean of an additional three baselines taken 30 sessions after the initial baseline determinations.

The strain differences in choice bias were dependent on the increasing delay imposed on the large reward response as evidenced by the effects of the "zero-delay" probe manipulation (in which both small and large reward responses were reinforced immediately). As shown in Figure 3C, all four strains of mice were equally responsive to this manipulation, maintaining a preference for the large reward response across the entire session. Under zero-delay conditions, there were no strain differences in either stable performance levels ( $\operatorname{strain} ; F_{(3,135)}=1.32 ; \mathrm{NS}$ ) or the time taken to reach the new baseline (c.6 sessions; strain; $F_{(3,25)}=$ 1.3 ; NS). The zero-delay data did show evidence of a slight general reduction (c.15\%) in choice of the large reward in the last block of trials, corresponding to where in the ascending delay condition the mice would be experiencing the longest $(8 \mathrm{sec})$ delay on the large reward response. However, the possible satiation effects indicated by these data did not influence the between-strain differences in choice bias because the pattern of responding at the $8 \mathrm{sec}$ delay was the same whether this block of trials was at the end or in a reversed delay manipulation (Fig. $3 D$ ) at the beginning of the session. Under the reversed delay conditions (i.e., 8, 4, 2, 1, and 0 $\mathrm{sec})$, the overall direction of choice bias was, as anticipated, reversed (delay; $F_{(4,135)}=3.2 ; p<0.02$ ), but the relative pattern of choice bias was the same as that observed previously in the ascending delay condition (strain $\times$ delay $\times$ direction; $F_{(12,270)}=$ 0.3 ; NS).

The variability in choice bias in the ascending delay condition was not related to differences between the strains in experiencing the "forced" (information) trial contingencies, because in the forced trials (in which no choice was available), all four strains made equal responses to the large and small reward-related stimuli at all delays (strain $\times$ choice $\times$ delay; $F_{(12,270)}=0.74$; NS; data not shown). The pattern of choice bias also appeared to be unrelated to behavior in other, more general, aspects of the task. All four groups of mice showed a high degree of stimulus control in performing the task as indexed by the rapid latencies to make a
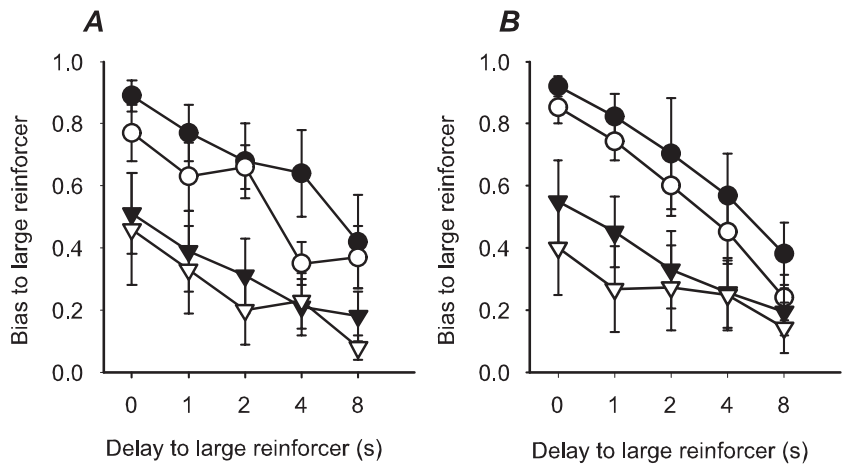

C

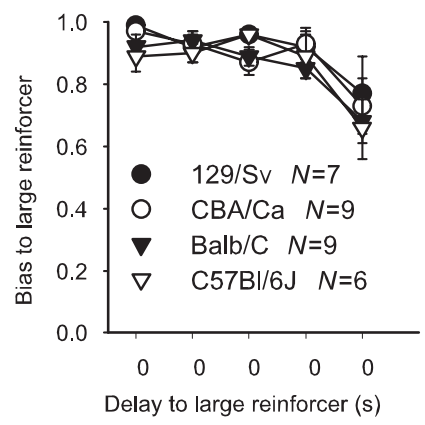

D

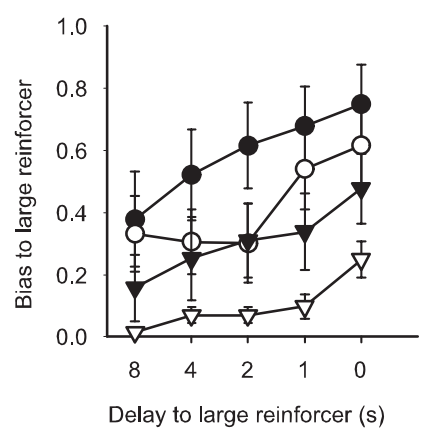

Figure 3. Choice response bias in the delayed-reinforcement task across strains. A, Graph showing change in choice bias with increasing delay in the delayed-reinforcement task for the four inbred strains of mice. A choice bias value of 1.0 would correspond to $100 \%$ choice of the large reward response. Data represent the mean \pm SEM of three consecutive sessions after initial acquisition of stable baseline performance (see Materials and Methods). All groups showed a change in choice bias away from the response leading to the delayed/large reinforcer toward the immediate/smaller reinforcer with increasing delay. There were systematic group differences in the choice bias function, indicative of strain-dependent variability in impulsive choice. Tukey's post hoc tests confirmed the following, in terms of impulsive choice: $(57 \mathrm{BL} / 6 \mathrm{~J}=$ $B A L B / C>129 / S v=C B A / C a$. B , Graph illustrating the stability of the strain effects on choice bias throughout the experiment. Data show the mean \pm SEM of three consecutive baseline sessions taken toward the end of the study, $\sim 30$ sessions after the data shown in A. C, Choice bias behavior under conditions in which no delay was imposed on the large reward response across the session. All strains showed a common shift upward in response bias, indicating a continued preference for the large reward response. Data represent the mean \pm SEM of three consecutive sessions when behavior was stable under the zero-delay condition. Note that these data were still divided into five separate delay blocks, although in the zero-delay condition there was no delay associated with the large reinforcer throughout the session. D, Reversal of the order of delays produced a reversed pattern of choice bias, but the pattern of strain differences remained the same. Data represent the mean $\pm S E M$ of three consecutive sessions when behavior was stable under the reversed delay condition.

center nose-poke at the start of a trial (start latency) (Fig. 4A) and then make a discriminative response (choice latency) (Fig. 4B). Moreover, the latency data were indifferent, across all strains, to the nature of the choice (small/immediate or larger/delayed; no main effect of choice on start latency: $F_{(1,223)}=3.56$, NS; no main effect of choice on choice latency: $F_{(1,223)}=0.15$, NS). There were main effects of strain in both the start latency $\left(F_{(3,135)}=27.52\right.$; $p<0.001)$ and choice latency $\left(F_{(3,135)}=12.94 ; p<0.001\right)$ data; post hoc tests (Tukey's) revealed that these were attributable, predominantly, in both cases, to generally slower responding in the $\mathrm{CBA} / \mathrm{Ca}$ mice. Tukey's post hoc tests showed that CBA/Ca mice were also distinct in terms of a relatively greater increase in nonstarted trials with increasing delay (Fig. 4C) (interaction between strain and delay; $\left.F_{(12,135)}=11.28 ; p<0.001\right)$ and a slightly higher rate of what were extremely rare (overall grand mean, 0.032 per session), omitted responses (main effect of strain; $\mathrm{F}_{(3,135)}=4.86$; 

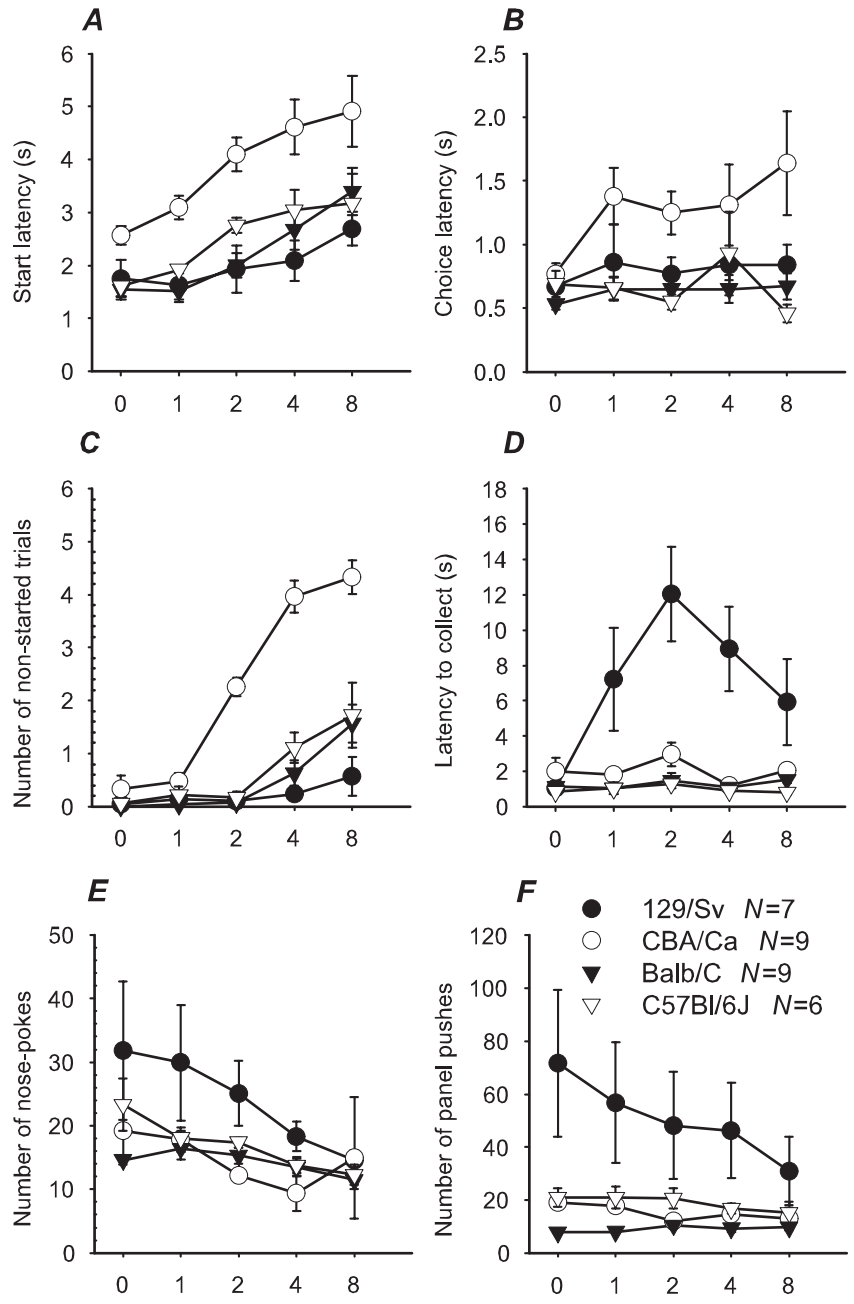

Delay to large reinforcer (s)

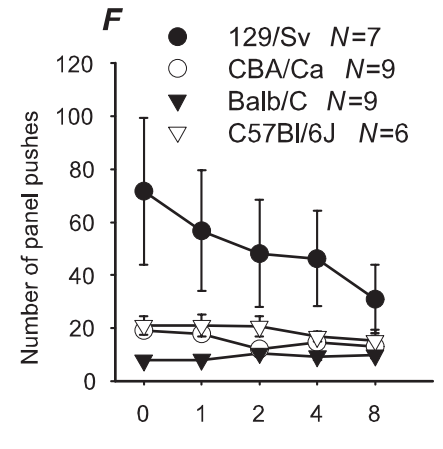

Delay to large reinforcer (s)

Figure 4. Strain differences in other behavioral measures in the delayed-reinforcement task. Graphs showing mouse strain performance on different aspects of the delayed-reinforcement task during choice trials (data from the same three baseline sessions as in Fig. 3A. A, Start latency (time taken to respond to the center light); $B$, choice latency (time taken to make choice response); $C$, number of non-started trials; $D$, latency to collect the reinforcer; $E$, total number of nose-pokes throughout a trial; $F$, total number of pushes on the food-hopper door (panelpushes). Data shown are means \pm SEM.

$p<0.01$; data not shown). Again though, behavior in these aspects of the task did not appear to be related to discriminative choice bias in any simple correlative manner, a conclusion that could be extended to other nonspecific measures in which, this time, post hoc (Tukey's) tests showed that the 129/Sv strain showed general differences to the other three strains. These included the time taken by the four strains to collect the reinforcer (Fig. $4 D$ ) (main effect of strain; $F_{(4,135)}=7.7 ; p<0.001$ ) and the pattern of nose-pokes and panel pushes made across the session (Fig. $4 E, F$ ) (main effect of strain on nose-pokes: $F_{(3,135)}=5.12$, $p<0.01$; main effect of strain on panel pushes: $F_{(3,135)}=9.71$, $p<0.001)$. Finally, there were no strain differences in the time taken by the mice to consume the reinforcer (overall grand mean, $5.3 \mathrm{sec}$; main effect of strain; $F_{(3,135)}=0.33$; NS; data not shown).

\section{Strain differences in spontaneous locomotor activity}

Activity scores for the four inbred lines, determined over 3 successive days, are shown in Figure 5. There were main effects of strain $\left(F_{(3,81)}=113.49 ; p<0.001\right)$ in the data, consistent with

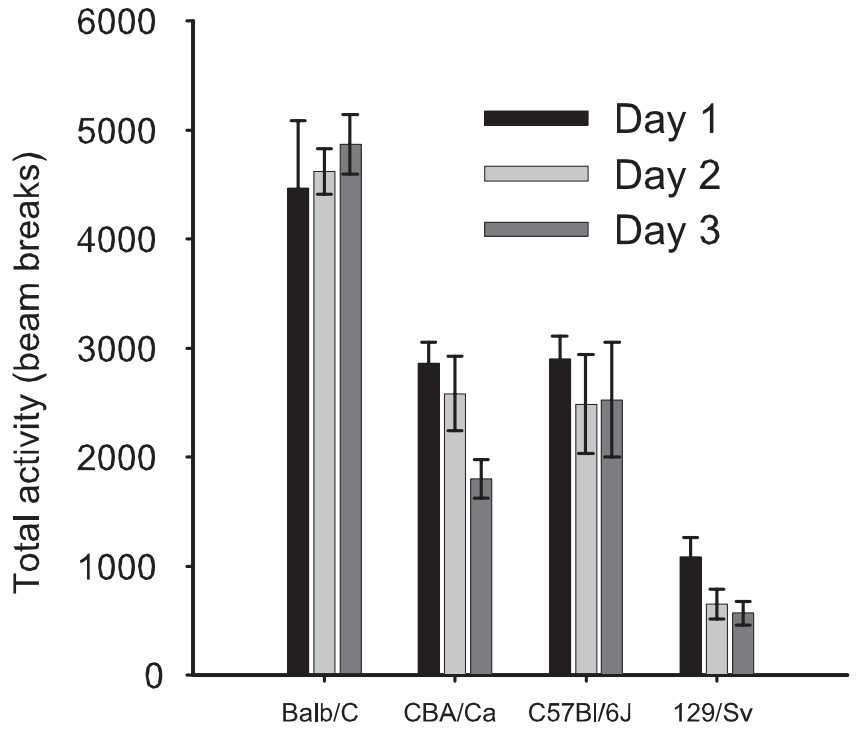

Figure 5. Locomotor activity levels in different strains. Graphs showing total locomotor activity (number of beam breaks) measured in activity cages in $2 \mathrm{hr}$ sessions, assessed on 3 consecutive days. Tukey's post hoc tests confirmed the following, in terms of locomotor activity levels: $\mathrm{BALB} / \mathrm{C}>\mathrm{C} 57 \mathrm{BL} / 6 \mathrm{~J}=\mathrm{CBA} / \mathrm{Ca}>129 /$ Sv. Data shown are means $\pm \mathrm{SEM}$.

Table 1. Heritability estimates for impulsivity and locomotor activity

\begin{tabular}{llll}
\hline & & \multicolumn{2}{l}{$90 \%$ Confidence limits } \\
\cline { 3 - 4 } Behavioral measure & Heritability & Non-central $F$ & Monte Carlo \\
\hline Average bias across a session & 0.158 & $0.037,0.399$ & $0.031,0.399$ \\
Mean bias at longest delay (8 sec) & 0.165 & $0.042,0.406$ & $0.033,0.402$ \\
Locomotor activity (day 3) & 0.751 & $0.652,0.849$ & $0.654,0.850$ \\
\hline
\end{tabular}

The analysis was performed with two behavioral measures indexing impulsivity, the average choice bias across a session and the bias at the longest delay $(8 \mathrm{sec})$, and stable locomotor activity scores on day 3 of testing. Confidence limits (90\%) were calculated from both the non-central $F$ distribution and Monte Carlo simulation, and these limits do not embrace the null hypothesis value of zero heritability (see Materials and Methods).

significant differences between the groups in terms of overall activity levels. Post hoc tests revealed the following pattern: $\mathrm{BALB} / \mathrm{c}$ (most active) $>\mathrm{C} 57 \mathrm{BL} / 6 \mathrm{~J}=\mathrm{CBA} / \mathrm{Ca}>129 / \mathrm{Sv}$ (least active). Three of the strains (C57BL/6J, CBA/C, 129/Sv) showed evidence of habituation to the test environment, manifest as reductions in activity levels with repeated testing. In contrast, the $\mathrm{BALB} / \mathrm{c}$ mice displayed similar levels of activity across the $3 \mathrm{~d}$. This differential pattern of habituation was reflected by a near significant interaction between the factors strain and day $\left(F_{(6,81)}=1.87 ; p=0.096\right)$.

\section{Heritability and genetic correlation of choice bias and} locomotor activity

The heritability of a trait can be derived by the proportion of variability attributable to genetics (between groups segregated by genotypes) over the total variation in the population (Wehner et al., 2001). Because individual members of inbred strains are isogenic, they provide an excellent model system from which to analyze the heritability of a trait (Falconer and Mackay, 1996). Using the methods for generating heritability scores for traits from inbred strains described by Hegmann and Possidente (1981) (for additional details, see Materials and Methods), we were able to produce heritability estimates for the choice bias and locomotor activity data (Table 1) and estimates of genetic correlation between these two factors (Table 2). The estimate of genetic effects in the choice bias data derived from the average bias across a session was $\sim 15.8 \%$, a figure very close to that computed 
Table 2. Correlation between impulsivity measures and locomotor activity (genetic and nongenetic variability)

\begin{tabular}{|c|c|c|c|c|}
\hline \multirow[b]{2}{*}{ Impulsivity measure } & \multicolumn{2}{|c|}{ Between strain (genetic) } & \multicolumn{2}{|c|}{$\begin{array}{l}\text { Within strain } \\
\text { (environmental) }\end{array}$} \\
\hline & Correlation & $p$ & Correlation & $p$ \\
\hline Average bias across a session & -0.922 & $<0.01$ & 0.129 & NS \\
\hline Mean bias at longest delay ( $8 \mathrm{sec}$ ) & -0.696 & $<0.05$ & 0.097 & NS \\
\hline
\end{tabular}

Between-strain analysis (the genetic component of variation) revealed a significant (based on the fact that the top confidence limits do not embrace the null hypothesis value of zero correlation) negative correlation between increased choice of the large reward response (less impulsive choice) and locomotor activity measures. In contrast, the within-strain analysis (the nongenetic component of variation) revealed no such correlation.

when using the alternate index of impulsive choice of the mean bias values for the longest $8 \mathrm{sec}$ delay, $16.5 \%$. The genetic contribution to variability in locomotor activity was higher at $\sim 75 \%$. Covariance analysis revealed a significant between-strain (genetic) correlation between locomotor activity and both indices of impulsivity, with those animals demonstrating higher locomotor activity also showing evidence of more impulsive choice. In contrast, for the within-strain (nongenetic) variability there was no systematic relationship between activity levels and choice bias.

\section{Discussion}

The principal findings were that genetics can contribute to variability in both impulsive choice and activity and that the genetic elements predicted complex behavioral associations, such that mice showing high levels of impulsive choice were also more active. It is unlikely that the behavioral correlation was attributable to confounds arising from mundane commonalities in task requirements. First, the patterns of effects seen in the response bias data were highly specific and independent of any systematic effects on other aspects of task performance. Second, in previous work, we have shown dissociations between the effects of D-amphetamine on choice bias and locomotor activity that confirm the independence of behavior in the murine delayedreinforcement task from changes in activity levels per se (Isles et al., 2003). Instead, the data were consistent with the existence of common genetic factors influencing variation in both impulsivity and locomotor activity.

The majority of the between-strain variability in choice bias was manifest as a shift in responding for the adjusted, large reinforcer, across all delays. Consequently, it was important to confirm the sensitivity of the strain differences to the increasing delays imposed on the large reward response. This was done by introducing sessions in which both small and large reward responses were reinforced immediately. These data (Fig. 3C) showed clearly that when delay was no longer present, not only did the mice maintain a large preference for the large reward response across the whole session but, crucially, the differences in choice bias between the strains disappeared completely. Although this finding provided evidence that the contingency between delay and reward was a main controlling variable of behavior in the delayed-reinforcement task and that all the mice were equally sensitive to the contingency, the lack of a significant interaction between strain and delay in the ascending delay condition suggested complex influences on choice behavior, resulting in strain differences at the start of the session when both the small and large reward were available immediately.

The present study was careful to exclude the possibility that the differences in response bias were influenced by basic factors related to primary motivation and learning/retrieval of the task, which could, conceivably, give rise to general, delay-independent effects. Furthermore, the data showing an equivalent pattern of strain effects under the descending delay condition (Fig. 3D) similarly excluded subtle effects because of differential satiety. In delayed-reinforcement paradigms, it has been suggested that choice bias behavior is governed by three, interacting, psychological processes: the perceived value of the reward and the perceived length and aversive nature of the delay (Mazur and Vaughan, 1987; Rodriguez and Logue, 1988; Ho et al., 1999). Therefore, it may be that the observed behavioral difference between the strains was a consequence of underlying differences in one or all of these factors. One option was that the strain differences at zero delay were attributable to carryover effects whereby the imposition of delay on the large reward response led to the development of a form of conditioned place aversion (creating a bias away from the location associated with delay even when the delay was negligible). In this interpretation, the effects on delayed reinforcement would be related to underlying strain differences in the development/persistence of learned associations, an interesting outcome in itself (Cardinal et al., 2001; Winstanley et al., 2004). However, such effects, if they did occur, clearly did not lead to an inflexible bias in responding, as demonstrated by the continued sensitivity to delay, both within a baseline session and in response to the zero-delay probe manipulation. Moreover, any conditioned aversion did not transfer to the forced trials because there were no between-strain differences suggestive of avoidance of the location associated with the delayed response in this part of the task. Hence, any explanation of the data involving an explicit conditioned aversion would have to take into account both the sensitivity to delay and the specificity to the choice component of the task.

In previous work, we suggested that delay aversion, in general (as opposed to conditioned effects), may be a significant controlling factor in murine delayed-reinforcement tasks, as a result of the high levels of predation experienced by this species (Isles et al., 2003). In this regard, we do have independent findings obtained in the elevated plus maze (data not shown), suggesting that if delay aversion does indeed play a major role in explaining the present findings, it is unlikely to be related to global strain differences in fear reactivity because the pattern of data seen in the elevated plus maze did not predict choice behavior in the test of impulsivity.

The strain differences may also have been a result of differences in the sensitivity of behavior to the effects of delay or the difference in reinforcer amount. It is not possible, currently, to distinguish, absolutely, between these alternatives because both strain-related differences in reward perception (and hence reinforcer efficacy) and alterations in timing would predict systematic effects on choice bias. However, a contribution from reward processes mediating the sensitivity of the subject's behavior to the differences in reinforcement amount may be more consistent with the baseline strain differences seen at zero delay (Pitts and Febbo, 2004). Importantly, these putative effects would again have to be specific to the psychologies taxed during the delayedreinforcement task, because there were no differences in the reinforcer habituation/preferences test conducted before the main experiment. Therefore, overall, we suggest that notwithstanding the lack of interaction between strain and delay, the pattern of data were consistent with effects of mouse strain on aspects of impulsive choice. In this regard, although much previous work on impulsive responding does report the effects of manipulations in terms of interactions with delay, there is evidence extending from the effects of brain lesions and drugs in rodents (Cardinal et al., 2001; Isles et al., 2003; Liu et al., 2004) to studies of drug dependence in humans (Madden et al., 1997), in which effects are 
present at zero delay. Evidently, although controlling variables may differ between species, such delay independent effects are not specific to the present murine version of the delayedreinforcement paradigm.

Regardless of the precise psychological mechanisms giving rise to the strain effects on impulsive choice, the heritability analysis confirmed a genetic component to this aspect of behavior. This was also the case when the heritability analysis was applied to activity data obtained independently from the same animals, in which the results were generally consistent with previous findings (Kopp, 2001). However, for both behaviors, particularly with respect to choice bias, there was appreciable residual variance because of nongenetic factors. The source of this "environmental" contribution to the overall variability is unspecified. We anticipated from previous data (Robbins et al., 1996; Francis et al., 2003) that the early life environment may be an important determinant of adult behavior and minimized the effects of this key variable by cross fostering all the mice to common mothers. Therefore, presumably, our data were influenced by other environmental factors we did not control for, which could have included, for instance, rank position within cage groups. This raises an important point about the generality of the data obtained here, which is that whereas they revealed clear genetic effects on impulsivity and activity, they should not be taken as providing estimates of the absolute genetic contribution to the variance between individuals across all situations. Obviously, this will depend on interactions between background genetics and the particular environmental influences.

The genetic and environmental effects on behavior were qualitatively distinct, insofar as it was only the genetic component that gave rise to a positive correlation between more impulsive responding and activity levels. The lack of association with environmental effects is of particular interest in light of previous data showing dissociations between hyperactivity and aspects of selfcontrol in rats subjected to social isolation. In this early life manipulation, in which subjects are housed separately from weaning, the most robust behavioral effect in adult animals is an enduring hyperactivity (Wilkinson et al., 1994). However, this is not matched by increases in impulsive responding. Indeed, if anything, social isolates show evidence of increased self-control across a variety of situations including delayed reinforcement, five-choice serial reaction time task (Liu et al., 2001), and conditional rule learning (Jones et al., 1991). It would seem that there is no monolithic relationship between hyperactivity and impulsiveness and that the extent to which they coexist depends on complex interactions between genetic and environmental effects.

The predictive relationship between activity and impulsive choice attributable to genetics raises questions at both the behavioral and gene level. In terms of behavior, one option is that the choice bias and activity data were linked via redundant effects on some common psychology. Here, the obvious possibility of confounds attributable to basic task requirements has been eliminated. Similarly, general effects on factors such as fear reactivity (which could, potentially, influence both exploration of an activity cage and the willingness to wait for a reward) do not predict a positive correlation. In the absence of a converging psychology, the alternative possibility would be that the common genetic effects impact on activity and impulsive responding via parallel, but independent, routes.

The present results do not identify the specific genetic polymorphisms that may underlie the behavioral effects. Most work on the genetic substrates of impulsive phenotypes, in both humans and animal models, has focused on dopamine, with poly- morphisms of the DRD2 (Blum et al., 1995), DRD4 (Langley et al., 2004), and DRD5 (Lowe et al., 2004) receptor subtypes implicated in aspects of AD/HD and OCD. However, in previous work, we have shown qualitative dissociations between the effects of D-amphetamine on choice bias and locomotor activity (Isles et al., 2003) that would tend to exclude, in the present case, explanations based on dopamine. Another interesting set of candidate genes may be those related to 5-HT functioning. Evidence is accumulating that 5-HT is a key controlling variable in impulsive behaviors, spanning, impulsive action (Harrison et al., 1997; Winstanley et al., 2003a), impulsive choice (Mobini et al., 2000; Winstanley et al., 2003b), locomotor activity (Geyer, 1996), and aggression (Hen, 1996). Furthermore, 5-HT-related genes demonstrate polymorphic variation in mice and man (see www. informatics.jax.org/searches/polymorphism[lowen]form.shtml and www.ensembl.org/), although the mouse data are far from complete. As such, it might be of most immediate profit to examine the extent to which 5-HT-related loci differ between strains and whether this variability maps onto the behavioral phenotypes seen here. A similar approach may prove useful with respect to potential strain-dependent polymorphisms in the regulatory region of the gene coding for MAOA, which has been associated in humans with variability in impulsivity mediated by 5 -HT effects (Manuck et al., 2000).

In conclusion, our data provide strong evidence for the influence of natural genetic variability on aspects of impulsivity and activity, which, we suggest, reflects the common influence of (multi-) allelic polymorphisms that have become fixed in a given inbred population. The overall variability in behavior also contained a major environmental component, highlighting the complexity of predicting behavioral outcomes in individuals, as epitomized by the recent data of Caspi et al. (2002) examining links between early life environment, MAOA polymorphisms, and antisocial behavior. The extent to which the current data extend to other types of impulsive responding requires additional investigation, as does the extent to which the data have relevance across species. However, notwithstanding the fact that mice and men faced different selective pressures, the data obtained in this model system may usefully inform the human literature in which comorbidity of impulsivity and hyperactivity occurs in several pathological conditions (Sheppard et al., 1999).

\section{References}

Blum K, Sheridan PJ, Wood RC, Braverman ER, Chen TJ, Comings DE (1995) Dopamine D2 receptor gene variants: association and linkage studies in impulsive-addictive-compulsive behaviour. Pharmacogenetics 5:121-141.

Bouwknecht JA, Hijzen TH, van der Gugten J, Maes RA, Hen R, Olivier B (2001) Absence of 5-HT(1B) receptors is associated with impaired impulse control in male 5-HT(1B) knockout mice. Biol Psychiatry 49:557-568.

Brunner D, Hen R (1997) Insights into the neurobiology of impulsive behavior from serotonin receptor knockout mice. Ann NY Acad Sci 836:81-105.

Brunner HG, Nelen M, Breakefield XO, Ropers HH, van Oost BA (1993a) Abnormal behavior associated with a point mutation in the structural gene for monoamine oxidase A. Science 262:578-580.

Brunner HG, Nelen MR, van Zandvoort P, Abeling NG, van Gennip AH, Wolters EC, Kuiper MA, Ropers HH, van Oost BA (1993b) X-linked borderline mental retardation with prominent behavioral disturbance: phenotype, genetic localization, and evidence for disturbed monoamine metabolism. Am J Hum Genet 52:1032-1039.

Cardinal RN, Robbins TW, Everitt BJ (2000) The effects of D-amphetamine, chlordiazepoxide, alpha-flupenthixol and behavioural manipulations on choice of signalled and unsignalled delayed reinforcement in rats. Psychopharmacology (Berl) 152:362-375.

Cardinal RN, Pennicott DR, Sugathapala CL, Robbins TW, Everitt BJ (2001) 
Impulsive choice induced in rats by lesions of the nucleus accumbens core. Science 292:2499-2501.

Caspi A, McClay J, Moffitt TE, Mill J, Martin J, Craig IW, Taylor A, Poulton R (2002) Role of genotype in the cycle of violence in maltreated children. Science 297:851-854.

Chen CS, Burton M, Greenberger E, Dmitrieva J (1999) Population migration and the variation of dopamine $\mathrm{D} 4$ receptor (DRD4) allele frequencies around the globe. Evol Hum Behav 20:309-324.

Clark L, Robbins T (2002) Decision-making deficits in drug addiction. Trends Cogn Sci 6:361-363.

Ding YC, Chi HC, Grady DL, Morishima A, Kidd JR, Kidd KK, Flodman P, Spence MA, Schuck S, Swanson JM, Zhang YP, Moyzis RK (2002) Evidence of positive selection acting at the human dopamine receptor D4 gene locus. Proc Natl Acad Sci USA 99:309-314.

Evenden J (1999) Impulsivity: a discussion of clinical and experimental findings. J Psychopharmacol 13:180-192.

Evenden JL, Ryan CN (1996) The pharmacology of impulsive behaviour in rats: the effects of drugs on response choice with varying delays of reinforcement. Psychopharmacology (Berl) 128:161-170.

Falconer DS, Mackay TFC (1996) Introduction to quantitative genetics. Harlow, UK: Longman.

Francis DD, Szegda K, Campbell G, Martin WD, Insel TR (2003) Epigenetic sources of behavioral differences in mice. Nat Neurosci 6:445-446.

Geyer MA (1996) Serotonergic functions in arousal and motor activity. Behav Brain Res 73:31-35.

Harrison AA, Everitt BJ, Robbins TW (1997) Central 5-HT depletion enhances impulsive responding without affecting the accuracy of attentional performance: interactions with dopaminergic mechanisms. Psychopharmacology (Berl) 133:329-342.

Hegmann JP, Possidente B (1981) Estimating genetic correlations from inbred strains. Behav Genet 11:103-114.

Hen R (1996) Mean genes. Neuron 16:17-21.

Ho MY, Mobini S, Chiang TJ, Bradshaw CM, Szabadi E (1999) Theory and method in the quantitative analysis of "impulsive choice" behaviour: implications for psychopharmacology. Psychopharmacology (Berl) 146:362-372.

Humby T, Laird FM, Davies W, Wilkinson LS (1999) Visuospatial attentional functioning in mice: interactions between cholinergic manipulations and genotype. Eur J Neurosci 11:2813-2823.

Isles AR, Humby T, Wilkinson LS (2003) Measuring impulsivity in mice using a novel operant delayed reinforcement task: effects of behavioural manipulations and D-amphetamine. Psychopharmacology (Berl) 170:376-382.

Jones GH, Marsden CA, Robbins TW (1991) Behavioural rigidity and rulelearning deficits following isolation-rearing in the rat: neurochemical correlates. Behav Brain Res 43:35-50.

Kopp C (2001) Locomotor activity rhythm in inbred strains of mice: implications for behavioural studies. Behav Brain Res 125:93-96.

Langley K, Marshall L, van den Bree M, Thomas H, Owen M, O’Donovan M, Thapar A (2004) Association of the dopamine D4 receptor gene 7-repeat allele with neuropsychological test performance of children with ADHD. Am J Psychiatry 161:133-138.

Liu YP, Robbins TW, Wilkinson LS (2001) Attention, impulse control and hyperactivity in social isolates. J Psychopharmacol 15:A62.

Liu YP, Wilkinson LS, Robbins TW (2004) Effects of acute and chronic buspirone on impulsive choice and efflux of 5-HT and dopamine in hippocampus, nucleus accumbens and prefrontal cortex. Psychopharmacology (Berl) 173:175-185.

Lowe N, Kirley A, Hawi Z, Sham P, Wickham H, Kratochvil CJ, Smith SD, Lee SY, Levy F, Kent L, Middle F, Rohde LA, Roman T, Tahir E, Yazgan Y, Asherson P, Mill J, Thapar A, Payton A, Todd RD, et al. (2004) Joint analysis of the DRD5 marker concludes association with attention-deficit/hyperactivity disorder confined to the predominantly inattentive and combined subtypes. Am J Hum Genet 74:348-356.

Madden GJ, Petry NM, Badger GJ, Bickel WK (1997) Impulsive and self- control choices in opioid-dependent patients and non-drug-using control participants: drug and monetary rewards. Exp Clin Psychopharmacol $5: 256-262$

Manuck SB, Flory JD, Ferrell RE, Mann JJ, Muldoon MF (2000) A regulatory polymorphism of the monoamine oxidase-A gene may be associated with variability in aggression, impulsivity, and central nervous system serotonergic responsivity. Psychiatry Res 95:9-23.

Mazur JE, Vaughan Jr W (1987) Molar optimization versus delayed reinforcement as explanations of choice between fixed-ratio and progressiveratio schedules. J Exp Anal Behav 48:251-261.

Mobini S, Chiang TJ, Ho MY, Bradshaw CM, Szabadi E (2000) Effects of central 5-hydroxytryptamine depletion on sensitivity to delayed and probabilistic reinforcement. Psychopharmacology (Berl) 152:390-397.

Mobini S, Body S, Ho MY, Bradshaw CM, Szabadi E, Deakin JF, Anderson IM (2002) Effects of lesions of the orbitofrontal cortex on sensitivity to delayed and probabilistic reinforcement. Psychopharmacology (Berl) 160:290-298.

Pattij T, Broersen LM, van der Linde J, Groenink L, van der Gugten J, Maes RA, Olivier B (2003) Operant learning and differential-reinforcementof-low-rate 36-s responding in 5-HT1A and 5-HT1B receptor knockout mice. Behav Brain Res 141:137-145.

Pearson E, Tiku ML (1970) Some notes on the relationship between the distribution of central and non-central F. Biometrika 57:175.

Petry NM (2001) Pathological gamblers, with and without substance use disorders, discount delayed rewards at high rates. J Abnorm Psychol 110:482-487.

Pitts RC, Febbo SM (2004) Quantitative analyses of methamphetamine's effects on self-control choices: implications for elucidating behavioral mechanisms of drug action. Behav Process 66:213-233.

Robbins T, Jones G, Wilkinson L (1996) Behavioural and neurochemical effects of early social deprivation in the rat. J Psychopharmacol 10:39-47.

Rodriguez ML, Logue AW (1988) Adjusting delay to reinforcement: comparing choice in pigeons and humans. J Exp Psychol Anim Behav Process 14:105-117.

Sheppard DM, Bradshaw JL, Purcell R, Pantelis C (1999) Tourette's and comorbid syndromes: obsessive compulsive and attention deficit hyperactivity disorder. A common etiology? Clin Psychol Rev 19:531-552.

Sonuga-Barke EJ, Taylor E, Sembi S, Smith J (1992) Hyperactivity and delay aversion. I. The effect of delay on choice. J Child Psychol Psychiatry 33:387-398.

Stuart A, Ord JK (1991) Kendall's advanced theory of statistics. London: Arnold.

Swanson J, Posner M, Fusella J, Wasdell M, Sommer T, Fan J (2001) Genes and attention deficit hyperactivity disorder. Curr Psychiatry Rep 3:92-100.

Wehner JM, Radcliffe RA, Bowers BJ (2001) Quantitative genetics and mouse behavior. Annu Rev Neurosci 24:845-867.

Wilkinson LS, Killcross SS, Humby T, Hall FS, Geyer MA, Robbins TW (1994) Social isolation in the rat produces developmentally specific deficits in prepulse inhibition of the acoustic startle response without disrupting latent inhibition. Neuropsychopharmacology 10:61-72.

Winstanley CA, Chudasama Y, Dalley JW, Theobald DE, Glennon JC, Robbins TW (2003a) Intra-prefrontal 8-OH-DPAT and M100907 improve visuospatial attention and decrease impulsivity on the five-choice serial reaction time task in rats. Psychopharmacology (Berl) 167:304-314.

Winstanley CA, Dalley JW, Theobald DE, Robbins TW (2003b) Global 5-HT depletion attenuates the ability of amphetamine to decrease impulsive choice on a delay-discounting task in rats. Psychopharmacology (Berl) 170:320-331.

Winstanley CA, Theobald DE, Cardinal RN, Robbins TW (2004) Contrasting roles of basolateral amygdala and orbitofrontal cortex in impulsive choice. J Neurosci 24:4718-4722. 